\title{
Einflussfaktoren bei der Social-Media-Nutzung in der Wissenschaftskommunikation
}

\author{
Anne Hennig • Sarah Kohler
}

Online publiziert: 15 . Oktober 2020

(C) Der/die Autor(en) 2020

Zusammenfassung Social Media beherrschen nicht nur die private Kommunikation, sondern haben zunehmend auch Einfluss auf die externe Wissenschaftskommunikation. In bisherigen Forschungsarbeiten wurden zwar bereits Social Media und deren Nutzung durch Wissenschaftler*innen in Zusammenhang mit wissenschaftsexterner Kommunikation untersucht, allerdings mangelt es an einer systematischen Darstellung der Einflussfaktoren. Diese Arbeit möchte an vorangegangene Studien anknüpfen und die gefundenen Einflussfaktoren replizieren und verifizieren. Als maßgebliche Einflussfaktoren bei der Social-Media-Nutzung von Wissenschaftler*innen haben wir basierend auf dem Forschungsstand Disziplinzugehörigkeit, den beruflichen Kontext, persönliche Eigenschaften und Prädispositionen definiert. Untersucht wurde die Häufigkeit der Nutzung von Facebook, Twitter, Instagram, YouTube, Snapchat sowie von Blogs und Podcasts. In einer aufwändig angelegten Online-Befragung haben wir zunächst Wissenschaftler*innen von 21 deutschen Volluniversitäten zur Teilnahme an der Studie eingeladen. Die Rekrutierung erfolgte über Dekanate und Institute und führte zu einer Stichprobe von über 1100 Wissenschaftler*innen. Lediglich Facebook und Twitter erwiesen sich für die Kommunikation im wissenschaftsexternen Kontext als relevant. Nach einer binär-logistischen Regressionsanalyse zeigte sich, dass die Ergebnisse bisheriger Studien nur vereinzelt bestätigt werden konnte. Faktoren wie Disziplinzugehörigkeit, Qualifikationsstufe und Alter

Die Ergebnisse der vorliegenden Arbeit sind Auszüge der Abschlussarbeit von Anne Hennig im Master Wissenschaft - Medien - Kommunikation am Institut für Technikzukünfte, Department für Wissenschaftskommunikation, des Karlsruher Instituts für Technologie.

\footnotetext{
A. Hennig, M.A. $(\bowtie) \cdot$ Dr. phil. S. Kohler

Department für Wissenschaftskommunikation, Institut für Technikzukünfte, Karlsruher Institut für Technologie, Englerstraße 2, 76131 Karlsruhe, Deutschland

E-Mail: anne.hennig@posteo.de

Dr. phil. S. Kohler

E-Mail: sarah.kohler@kit.edu
} 
haben eine nur mäßig gute Erklärkraft. Der Social-Media-Typus der Hochschule, das Geschlecht, Technikaffinität oder -abneigung sowie Persönlichkeitsfaktoren erwiesen sich als nicht signifikant. Die eher geringen R-Quadrat-Werte in allen Modellen legen nahe, dass die unabhängigen Variablen nicht in der Lage sind, die abhängigen Variablen ausreichend gut zu erklären. Somit bedarf es weiterer Forschung, die insbesondere auch Faktoren des Einflusses auf das Public Engagement von Wissenschaftler*innen unabhängig vom Kommunikationsmedium einbezieht.

Schlüsselwörter Externe Wissenschaftskommunikation · Public Engagement · Social-Media-Nutzung · Twitter · Facebook · Partizipation · Planned Behavior

\section{Impacts on the communication of scientists in social media}

Abstract Social media networks have as much impact on science communication as they have on our daily life. The use of social media for science communication purposes has already been investigated within several studies. But there is a lack of systematic research on factors which describe scientists' use of social media for external science communication.

Science communication in this paper is defined as the use of different methods and media to communicate science. According to Burns et al., "science communication may involve science practitioners, mediators, and other members of the general public, either peer-to-peer or between groups" and is used to stimulate Awareness, Enjoyment, Interest, Opinions and Understanding to/for science (AEIOU definition). Science communication can be internal towards the scientific community, or external towards the public. Schäfer divides internal communication in formal (via journals, conferences) or informal communication (face-to-face communication, communication via e-mail or social media). External science communication is directed at persons outside the scientific community and can be mediated by journalists, communication experts or scientists themselves (self-mediated external science communication).

This study ties in with previous research and examines factors which were described as decisive for the use of social network in the context of self-mediated external science communication: affiliation (area of subject, application relevance of the research as well as competitiveness of the research area), professional context (career level, social media type of the university), personal characteristics (age, gender), and predispositions (personality, curiosity about new technological developments, computer anxiety, privacy concerns). While there has not been an established theoretical approach to this topic yet, the theory of planned behavior (TPB) is used to explain the motivations of scientists for using social media for their communication about science. Attitude, subjective norms and perceived behavioral control have been described as motivations which impact the intention to perform a certain behavior. Researching the influencing factors helps to better understand researchers' intentions for communicating science in social networks. The concise description of influencing factors and the analysis of these factors closes a gap in the research on scientists' use of social media. 
In this study, social media use was defined as the frequency of using Facebook, Twitter, Instagram, YouTube, Snapchat as well as blogs and podcasts for private or work-related communication as well as external science communication. Platforms like ResearchGate, Academia, LinkedIn or Xing were not analyzed. The networks have been described as not relevant for external science communication and are rather used for internal communication with own peers.

The questionnaire was designed as an online survey and implemented with the German survey tool SoSciSurvey. In an extensive sampling process, 21 German universities were chosen to retrieve a diverse sample of scientists. A non-probability sampling method with purposive sampling was used to define the universities. A sample of scientists was recruited by contacting deaneries and institutes via email. These institutions were then asked to forward the invitation link to their research staff. It was not the aim to draw a representative sample, because this method does not allow to relate which persons did in fact get the invitation link. The main focus was to reach as many scientists with different backgrounds as possible. In the end, over 1100 scientists completed the questionnaire. Students, trainees or technical as well as non-academic staff were excluded from the analyzed data.

At first, the participants specified whether they use a social network daily, weekly, monthly, less than monthly or not at all. Persons, who stated that they use a certain social media at least sometimes, were asked to specify whether they use this social network for private communication, for the search for information, for the communication with colleagues, other scientists or students or for the communication with layperson and external experts like journalists, politicians etc. Participants who stated that they do not use a certain social network were asked to name the main reason for not using it. The analysis showed that only Facebook and Twitter are relevant for communication in a professional and scientific context. YouTube, blogs and podcasts were mainly used for the search for information and barely within the context of science communication. It is likely that the production of video- and audiocontent is too time-consuming for most of the researchers. Although blogs are a nice way to disseminate own research publicly as well as in the scientific community, blogs need constant maintenance to be visible. Snapchat was only used for private use and at least four percent of the respondents used Instagram to communicate with layperson about science.

By means of a binary logistic regression it could further be found that results from previous research were hardly confirmed. Factors like affiliation, age, or career level — which used to be important in other studies - had few influence. The results showed that scientists who define their research area as rather competitive, are more likely to communicate about science on Twitter or Facebook. A researcher's career level just proofed to be impactful with Twitter users and the career stages "junior professor" or "associate professor" in the context of work-related communication. Age had an impact on the use of Facebook: Older persons were more likely to use Facebook for external science communication. Contrary to expectations there was no significant impact detected fort the area of subject, application relevance of the research, social media type of the university, gender, personality, curiosity about new technological developments, computer anxiety or privacy concerns. 
In all regression models, Nagelkerke $\mathrm{R}^{2}$ was rather low with values between 0.166 (use of Facebook for work-related science communication) and 0.229 (use of Twitter for work-related science communication). This indicates that the explanatory variables were not sufficient in describing the explained variables. Further research is needed to draw a more detailed picture on the use and non-use of social media in the self-mediated external science communication. It could be promising to further analyze correlations between the influencing factors and the reasons why scientists are not using social media for external communication, for example. Either way, the influence of factors related to public engagement of activities of scientists should be included.

Even if this study is rather limited in its explanatory power and further research is demanded, important information is delivered to understand the complex and multilayered interactions between science, society and the media in times of rapid technological changes.

Keywords Science communication - Social Media use · Public engagement · Facebook $\cdot$ Twitter $\cdot$ Scientist participation $\cdot$ Theory of planned behavior

\section{Einleitung}

Social Media beherrschen nicht nur die private Kommunikation, sondern haben zunehmend auch Einfluss auf die wissenschaftliche Kommunikation. „Das Internet und mit ihm die sogenannten Social Media haben die öffentliche, private und politische Kommunikation wie auch die Wissenschaftskommunikation revolutioniert", verlautbart die Deutsche Akademie der Technikwissenschaften in einer Stellungnahme zu Social Media und digitaler Wissenschaftskommunikation (acatech - Deutsche Akademie der Technikwissenschaften et al. 2017, S. 6). Revolutioniert wurden dabei nicht nur die Kommunikationsart - die im Sinne des Public Understanding of Science/Public Engagement of Science partizipativer und dialogischer geworden ist -, sondern vor allem auch die Möglichkeiten der Beteiligung an Wissenschaftskommunikation und damit die Zusammensetzung der Kommunikationsakteure (vgl. acatech - Deutsche Akademie der Technikwissenschaften et al. 2017, S. 6). Der geringe zeitliche und finanzielle Aufwand (vgl. Könneker 2017, S. 455) sowie die Selbstthematisierung, ,ohne dem journalistischen Selektionsprozess der Massenmedien unterworfen zu sein“ (Metag und Schäfer 2017, S. 165), werden als Vorteile gesehen, mit Social Media öffentlich auf Wissenschaftsthemen aufmerksam zu machen und sie zu kommunizieren. Wissenschaftskommunikation ist nach der „AEIOU“-Definition von Burns et al. (2003, S. 191) ein Zusammenspiel verschiedener Kommunikationsformen und Medienformate, um „Awareness“, „Enjoyment“, „Interest“ „,Opinions“ und „Understanding“ für oder gegenüber Wissenschaft zu erzeugen. Sie kann dabei nach Schäfer (2017a) in interne und externe Wissenschaftskommunikation unterteilt werden. Interne Wissenschaftskommunikation beschreibt die Kommunikation innerhalb der Scientific Community, entweder formell (über Fachzeitschriften, Fachtagungen) oder informell über persönliche Gespräche, E-Mails oder auch Social Media. Externe Wissenschaftskommunikation 
richtet sich an Personen(gruppen) außerhalb der Scientific Community und kann fremd- oder selbstvermittelt sein. Bei der selbstvermittelten externen Wissenschaftskommunikation unterscheidet Schäfer (2017a) zwischen primär interessengeleiteter Kommunikation, wie zum Beispiel über PR-Abteilungen, und nicht primär interessengeleiteter Kommunikation. In letzterem Fall initiieren Wissenschaftler*innen selbst die Kommunikation als kommunikativen Selbstzweck (vgl. Schäfer 2017a). Während Wissenschaftler*innen Online-Medien für interne Wissenschaftskommunikation teilweise intensiv verwenden, ist die Nutzung von Online-Kanälen für die wissenschaftsexterne Kommunikation jedoch kaum verbreitet (vgl. Schäfer 2017b, S. 279). Verschiedene Forschungsarbeiten (vgl. Donk 2012; Pscheida et al. 2014; Lo 2016; Scheu und Schedifka 2018) befassten sich bereits mit möglichen Einflussfaktoren. Unsere Arbeit möchte daran anknüpfen und die gefundenen Einflussfaktoren für die wissenschaftsexterne Kommunikation in einer umfassend angelegten Studie replizieren und verifizieren.

\section{Außenkommunikation und Social-Media-Nutzung von Wissenschaftler*innen}

Um sinnvolle Strategien für eine nachhaltige Nutzung von Social Media durch Wissenschaftler*innen in der externen Wissenschaftskommunikation zu erarbeiten, ist es wichtig, Einflussfaktoren bei der Social-Media-Nutzung zu kennen und quantifizieren zu können. Ähnlich wie bei der Online-Kommunikation von Hochschulen (vgl. Metag und Schäfer 2017, S. 168) liegt auch für Einflüsse auf die individuelle Wissenschaftskommunikation von Wissenschaftler*innen in den Social Media bisher noch kein etabliertes theoretisches Modell vor. Die Forschung hat sich der Thematik bisher zwar qualitativ angenähert, aber die Einflussfaktoren noch nicht quantitativ und systematisch erfasst.

Betrachtet man Motivationsfaktoren für die Außenkommunikation von Wissenschaftler*innen im Allgemeinen, erweist sich die Theory of Planned Behavior (TPB) als passendes Modell (vgl. z. B. Poliakoff und Webb 2016; Dudo und Besley 2016; Besley et al. 2018). Die aus der Psychologie stammende Theorie des geplanten Verhaltens beschreibt ein Modell zur Vorhersage von Verhalten und ist eine Weiterentwicklung der Theorie of Reasoned Action (TRA). „In fact, the theory of planned behavior differs from the theory of reasoned action in its addition of perceived behavioral control.“ (Ajzen 1991, S. 183) Während es logisch erscheint, dass reale, mehr oder weniger selbst kontrollierbare Faktoren (z.B. Ressourcen wie Zeit, Geld oder Fähigkeiten) das Verhalten beeinflussen, wurde mit der TPB zusätzlich der Einfluss der wahrgenommen Verhaltenskontrolle beschreiben. Die Einstellung gegenüber einem Verhalten sowie subjektive Normen und die wahrgenommene Verhaltenskontrolle erklären die Motivation, die hinter einer Verhaltensweise steht. Die Faktoren beeinflussen sich dabei gegenseitig. Die wahrgenommene Verhaltenskontrolle kann zudem direkt auf die Verhaltensweise Einfluss nehmen. Im Allgemeinen ist ein Verhalten der Theorie zufolge umso wahrscheinlicher, je stärker die Motivation für und/oder je stärker die wahrgenommene Kontrolle über das Verhalten ist (vgl. Ajzen 1991, S. 181-184). 
Als Motivationsfaktoren für Außenkommunikation im Allgemeinen werden zum Beispiel Legitimationsgründe genannt: Wissenschaft verteidigen, informieren, begeistern oder Vertrauen schaffen (vgl. Dudo und Besley 2016), Kompetenz beweisen, Transparenz herstellen oder Werte vermitteln (vgl. Besley et al. 2018) sowie Inhalte verbreiten, Verbesserungen herbeiführen oder Rechtfertigung der Ausgabe öffentlicher Gelder (vgl. Scheu und Schedifka 2018). Schäfer (2017b, S. 279) verweist im Bereich der Online-Kommunikation zusätzlich auf die Motivation, falsche, pseudowissenschaftliche oder einseitig interpretierte Informationen richtigstellen zu wollen. In Bezug auf öffentliche Kommunikation sehen Wissenschaftler*innen sich selbst daher auch als Mediatoren, Multiplikatoren oder Gatekeeper (vgl. Jünger und Fähnrich 2020, S. 391).

Auch die Außenkommunikation von Wissenschaftler*innen in unterschiedlichen digitalen Kommunikationskanälen wurde bereits in einigen Studien untersucht. Allerdings fehlt bisher eine systematische Darstellung der Einflussfaktoren bei der Social-Media-Nutzung von Wissenschaftler*innen unter Berücksichtigung einer klar definierten Menge an Social-Media-Kanälen. Schäfer (2017b) stellte fest, dass die vielfältigen Studien zur Nutzung von Online-Kommunikation von Wissenschaftler*innen nur „,begrenzt vergleich- oder generalisierbar“ (Schäfer 2017b, S. 278) sind. Gründe hierfür sind unterschiedliche Definitionen von Social Media sowie starke Fluktuationen im Bereich der „,neuen“ oder „digitalen“ Medien. Beispielsweise zählen Bader et al. (2012) Mailinglisten und Blogs zu den digitalen Kommunikationsmedien, während aktuellere Studien (vgl. Scheu und Schedifka 2018) hauptsächlich Social Media wie Twitter, Facebook etc. einbeziehen. Konnten im Jahr 2010 noch StudiVZ oder MySpace (vgl. Perez 2010) oder im Jahr 2014 noch Google+ (vgl. Pscheida et al. 2014) zu den Social Media gezählt werden, waren diese bereits wenige Jahre später nicht mehr am Markt.

Je nach Plattform, ob Facebook, Twitter, Blogs, YouTube oder andere, gibt es eigene Präferenzen bei Nutzungsmotivation und Anwendungen. So werden in der internen Wissenschaftskommunikation Social Media am häufigsten für Kommunikation und den Austausch von Informationen genutzt, während Weblogs, Mikroblogs oder auch Facebook hauptsächlich zu Recherchezwecken hinzugezogen werden (vgl. Collins et al. 2016). Für die externe Wissenschaftskommunikation sind Social Media wie Web- und Mikroblogs sowie Video- und Foto-Community-Portale laut Pscheida et al. (2014, S. 27) weniger relevant. Es zeigt sich auch, dass Plattformen wie Facebook, die nicht primär auf wissenschaftliche, sondern private Kommunikation ausgerichtet sind (vgl. Pscheida et al. 2014; Collins et al. 2016), häufig nur dann für Wissenschaftskommunikation genutzt werden, wenn sie auch im beruflichen und akademischen Kontext Anwendung finden (vgl. Pscheida et al. 2014, S. 31). Für interne Wissenschaftskommunikation wird dann eher auf ResearchGate, Academia oder Mendeley zurückgegriffen (vgl. Focke 2017, S. 215). Twitter wird in der wissenschaftsinternen Kommunikation vor allem genutzt, um eigene oder andere Forschungsergebnisse publik zu machen, über Konferenzen zu berichten oder sich mit anderen Wissenschaftler*innen auszutauschen (vgl. Collins et al. 2016; Rauchfleisch 2017; Jünger und Fähnrich 2020). Dabei verbleibt die öffentlichkeitswirksame Kommunikation von Wissenschaftler*innen auf Twitter trotz der den Social Media inhärenten dialogischen und interaktiven Ausrichtung stark unidirektional (vgl. Davies 
und Hara 2017, S. 565). YouTube ist in der internen Wissenschaftskommunikation kaum verbreitet. Der Zeit- und Arbeitsaufwand für die Produktion von Videos ist hierfür vermutlich ausschlaggebend (vgl. Geipel 2018, S. 191). Wissenschafts-Blogs hingegen haben eine Doppelrolle in der Wissenschaftskommunikation, da Wissenschaftler*innen mit Blogs sowohl ihre Forschungen der Öffentlichkeit präsentieren und zu Diskussionen anregen, als auch ihre Forschungsergebnisse wissenschaftsintern publik machen können (vgl. Mahrt und Puschmann 2014, S. 6). Entsprechend populär ist diese Form der digitalen Wissenschaftskommunikation, auch wenn aufgrund von Zeitmangel relativ wenige Wissenschaftler*innen selbst bloggen (vgl. Bonetta 2007, S. 444). Es mangelt noch an fundierten Studien zur Nutzung von Instagram und Snapchat für die Wissenschaftskommunikation.

Obwohl Social Media insbesondere in der externen Wissenschaftskommunikation wenig genutzt werden, zeigte sich, dass Wissenschaftler*innen generell für die Nutzung von Social Media aufgeschlossen sind, wenig Berührungsängste haben (vgl. Pscheida et al. 2014, S. 45) und Soziale Medien als Kommunikationsmittel grundsätzlich positiv bewerten (vgl. Scheu und Schedifka 2018, S. 194). Gegen eine Nutzung von Social Media in der Wissenschaftskommunikation sprechen der bereits beschriebene Zeitaufwand, die als gering empfundene Nützlichkeit und die Sorge über einen möglichen Missbrauch persönlicher Daten (vgl. Bader et al. 2012; Pscheida et al. 2014; Schäfer 2017b).

\section{Einflussfaktoren bei der Social-Media-Nutzung}

Als Einflussfaktoren für die Nutzung spezieller digitaler Kommunikationsmedien werden zunächst Alter und Disziplinzugehörigkeit näher betrachtet (vgl. Donk 2012, S. 186). So zeigt sich zum Beispiel in Interviews, dass Wissenschaftler*innen aus Disziplinen, die durch eine gesellschaftlich ausgerichtete Sozialisation geprägt sind (zum Beispiel Geschichts- oder Erziehungswissenschaftler*innen bzw. Vertreter*innen der Volkswirtschaftslehre) Social Media eher aktiv und eher mit einer wissenschaftsexternen Ausrichtung nutzen als Wissenschaftler*innen anderer Disziplinen (vgl. Scheu und Schedifka 2018, S. 202-203).

Die Disziplinzugehörigkeit wird wiederum von dem konkreten Forschungsfeld (anwendungsorientierte Projekte sind eher Gegenstand der Kommunikation, wettbewerbsorientierte Forschungsfelder eher weniger), dem beruflichen Kontext (Wissenschaftler*innen auf einer niedrigeren Qualifikationsstufe nutzen Social Media vergleichsweise intensiver, vor allem zur Förderung ihrer Karrierechancen) und den persönlichen Eigenschaften (Technikaffinität und normative Einstellung zur Wissenschaftskommunikation) beeinflusst (vgl. Scheu und Schedifka 2018, S. 202-204).

Donk (2012, S. 192) weist darauf hin, dass von einer positiven Einstellung gegenüber Technik im Allgemeinen nicht gleichzeitig auf eine positive Einstellung gegenüber Social Media geschlossen werden kann. Daher gilt auch die Einstellung zu Social Media als Einflussfaktor. Pscheida et al. (2014, S. 45) resümieren, dass Wissenschaftler*innen sich überwiegend aufgeschlossen im Umgang mit Social-Media-Anwendungen zeigen und wenig Berührungsängste haben - während sie vor allem Datenschutzaspekte als Gründe für die Nichtnutzung online-basierter An- 
wendungen angeben. In Hinblick auf mögliche Korrelationen der Einflussfaktoren konnten die Autoren zeigen, dass Interesse an technischen Neuheiten im Allgemeinen zur Nutzung und Beschäftigung mit den Kommunikationsmitteln anregt (vgl. Pscheida et al. 2014, S. 46).

Auch generelle Persönlichkeitsprädispositionen zählen in Anlehnung an die Bedeutung der Persönlichkeitsfaktoren innerhalb der Theory of Planned Behavior als Einflussfaktoren. Correa et al. (2010, S. 251-252) konnten zeigen, dass Personen, die extrovertierter sind als andere, häufiger Social-Media-Anwendungen nutzen. Dies traf auch auf emotional instabile Personen sowie offenere bzw. kreativere und innovativere Personen zu (vgl. auch Ryan und Xenos 2011). Hughes et al. (2012) zeigten, dass Emotionalität bzw. Neurotizismus die Facebook-Nutzung für soziale Zwecke gut erklären konnte. Für die Facebook-Nutzung zum Zweck der Information hingegen spielen neben Neurotizismus auch Extraversion und Offenheit eine Rolle (vgl. Hughes et al. 2012, S. 566-567). Im Allgemeinen, so die Autoren, korrelierten Persönlichkeitsprädispositionen zwar mit der Nutzung von Sozialen Netzwerken, allerdings seien diese Korrelationen ,not straightforward or as influential as some previous research has suggested" (Hughes et al. 2012, S. 268). Zudem deuten die Ergebnisse darauf hin, dass es netzwerkspezifische Nutzungsunterschiede gibt, die auf weitere Einflussfaktoren neben den Persönlichkeitsprädispositionen verweisen. So zeigen Liu und Campbell (2017), dass der Einfluss der bisher als stark identifizierten Faktoren Extraversion und Offenheit wesentlich geringer sein könnte als erwartet. Die angenommene starke Korrelation scheine eher durch die Varianz zustande zu kommen, die Extraversion mit anderen Persönlichkeitsfaktoren teile: „Controlling for correlations between personality factors in regression analysis resulted in a significant drop in the strength of the association." (Liu und Campbell 2017, S. 235) Stattdessen vermuten die Autoren, dass durch reziproke Effekte der Social-Media-Nutzung bestimmte Persönlichkeitsmerkmale verstärkt werden (vgl. Liu und Campbell 2017, S. 237).

Wie schon erwähnt, zählt das Alter der Wissenschaftler*innen ebenfalls zu den Einflussfaktoren. „Respondents preferring to write for online media were significantly younger on average [...]. They were more likely in earlier stages of their career [...] and without a management position [...]", resümiert Lo (2016, S. 154). Eine Befragung unter Neurowissenschaftlern von Allgaier et al. (2013b, S. 77) zeigte zudem, dass viele Befragte ,zwar das Wirkungspotential der neuen Medien [sehen]“, diese aber selbst nicht intensiv nutzen. Unterschiede konnten die Autoren hier zu jüngeren sowie US-amerikanischen Wissenschaftler*innen feststellen. Diese beiden Gruppen nutzen ,neue“ Medien häufiger und schreiben ihnen auch ein stärkeres Wirkungspotenzial zu als deutsche und ältere Wissenschaftler*innen (vgl. Allgaier et al. 2013b, S. 77). Einen signifikanten Einfluss des Geschlechts auf Kommunikationspräferenzen in den Social Media konnten die genannten Autoren allerdings nicht feststellen. Hinweise geben Pscheida et al. (2014, S. 46): Die Autoren konnten zeigen, dass sich Wissenschaftler stärker mit neuen Technologien beschäftigen als Wissenschaftlerinnen.

In der Literatur wird der Tätigkeitsschwerpunkt (hauptsächlich Lehre oder hauptsächlich Forschung bzw. beides gleichermaßen) ebenfalls als Einflussfaktor untersucht. Bader et al. (2012, S. 13) weisen darauf hin, dass digitale Medien dann genutzt 
werden, ,wenn sich diese Nutzung in ihre schon etablierte wissenschaftliche Praxis einbetten lässt, d.h. zur (besseren) Lösung schon existierender kommunikativer Aufgaben beiträgt“" und ,die Nutzung auf erkennbare Art und Weise die wissenschaftliche Praxis effektiver macht und deren Möglichkeiten erweitert". Weiterhin zeigen Pscheida et al. (2014, S. 33), dass insbesondere jene Befragten, die sowohl in Forschung als auch in Lehre eingebunden sind, wenig experimentierfreudig in Hinblick auf „neue“ Kommunikationsformen sind, da sie dafür keine Zeit haben. Auch konnten die Autoren zeigen, dass onlinebasierte Tools eher für die Forschung relevant sind und insbesondere Soziale Netzwerke sowie Mikroblogs im Lehrkontext „eine stark untergeordnete Rolle“ (Pscheida et al. 2014, S. 30) spielen.

In Zusammenhang mit dem Einfluss der Hochschule auf die Social-Media-Nutzung von Wissenschaftler*innen ist die Studie von Metag und Schäfer (2017) interessant. Die Autoren identifizierten fünf verschiedene Hochschul-Kommunikationstypen: Social-Media-Spezialisten, Allrounder, Verweigerer, Traditionelle und Mainstream. Social-Media-Spezialisten sind im Vergleich zu allen anderen Hochschulen am stärksten in den Social Media präsent, während die „Verweigerer“ nur wenig Online-Kommunikation betreiben (vgl. Metag und Schäfer 2017, S. 177). Wie Maasen und Wenninger (2017, S. 310) darstellen, positionieren sich institutionell gut vernetzte Wissenschaftler*innen grundsätzlich positiv gegenüber der Social-MediaNutzung. Zudem konnten Friedrichsmeier et al. (2015, S. 146) zeigen, dass die öffentliche Hochschulkommunikation sich aus den Kommunikationsleistungen des Hochschulpersonals und der PR-Abteilungen der Hochschule zusammensetzt. Interessant ist daher in diesem Zusammenhang, ob die Social-Media-Aktivität der Hochschule die Kommunikation der dort beschäftigten Wissenschaftler*innen beeinflusst.

In Anlehnung an die beispielhafte Einteilung der Einflussfaktoren von Scheu und Schedifka (2018, S. 207) und unter Berücksichtigung der oben genannten weiteren möglichen Faktoren werden die Einflussfaktoren für die Social-Media-Nutzung von Wissenschaftlern folgendermaßen kategorisiert:

- Persönliche Eigenschaften der Befragten: Alter und Geschlecht (Soziodemografie), Big-Five-Persönlichkeitsfaktoren (Extraversion, Neurotizismus, Verträglichkeit, Offenheit, Gewissenhaftigkeit), Technikaffinität und -abneigung;

- Disziplinzugehörigkeit: Fachbereich, Anwendungsbezug und Kompetitivität des Forschungsfeldes;

- Beruflicher Kontext der Befragten: Qualifikationsstufe und Social-Media-Aktivität der Hochschule.

Replikation und Verifikation dieser Einflussfaktoren ist das Ziel der diesem Artikel zugrunde liegenden Forschungsarbeit. Dabei prüfen wir, wie Social Media von Wissenschaftler*innen genutzt werden und inwieweit die bereits erfassten Einflussfaktoren mit der Social-Media-Nutzung zum Zweck der Wissenschaftskommunikation korrelieren. Die grundlegende Forschungsfrage für diese Studie lautet daher: Wie nutzen Wissenschaftler*innen Social Media und welche Einflussfaktoren erklären die für Externe sichtbare Wissenschaftskommunikation von Wissenschaftler*innen in den Social Media? 


\section{Methode}

Als Erhebungsinstrument diente eine standardisierte Online-Befragung, die mit Hilfe des Tools SoSciSurvey konzipiert wurde. Der Fragebogen wurde sowohl in deutscher als auch in englischer Sprache verfasst, da Deutsch angesichts des teilweisen hohen Anteils ausländischer Doktorand*innen (zum Beispiel FU Berlin: 35\%; vgl. Freie Universität Berlin o.J.) nicht vorauszusetzen war. Mit einem funktionellen Pretest und einem Pretest nach dem Think-Aloud-Verfahren wurde der Fragebogen geprüft und im Anschluss angepasst.

\subsection{Operationalisierung}

\subsubsection{Nutzung von Social Media}

Im Rahmen dieser Arbeit wurde die nicht primär interessengeleitete, externe Wissenschaftskommunikation in den Social Media untersucht. Mit Rückbezug auf Scheu und Schedifka (2018) sowie Pscheida et al. (2014) gehörten dazu Facebook (vgl. Focke 2017), Twitter (vgl. Rauchfleisch 2017), Instagram (vgl. Scheu und Schedifka 2018), YouTube (vgl. Geipel 2017) und Snapchat (vgl. wissenschaftskommunikation.de o.J.) sowie Blogs (vgl. Bonetta 2007; Allgaier et al. 2013b; Mahrt und Puschmann 2014; Wenninger 2016) und Podcasts. Plattformen wie ResearchGate und Academia, aber auch LinkedIn oder Xing, die eher zur wissenschaftsinternen Kommunikation genutzt werden (vgl. Focke 2017), blieben unberücksichtigt.

In einem ersten Schritt fragten wir nach der Nutzungshäufigkeit (täglich, mehrfach wöchentlich, wöchentlich, monatlich, seltener, nie oder kenne ich nicht). Gaben die Befragten an, mindestens ,selten“ darauf zuzugreifen, sollten sie anschließend mit sieben Items den Nutzungszweck des jeweiligen Kanals näher definieren. Wie bereits erwähnt, geht es in dieser Auswertung primär um die Nutzung von Social Media für die wissenschaftsexterne Kommunikation bzw. um von Externen wahrnehmbare wissenschaftliche Kommunikation. Da in den Social Media allerdings die Grenzen zwischen privater, wissenschaftsinterner und wissenschaftsexterner Kommunikation verschwimmen, ist nach Jünger und Fähnrich (2020) davon auszugehen, dass die öffentliche Sichtbarkeit einer Wissenschaftlerin/eines Wissenschaftlers in den Social Media als Wissenschaftskommunikation im Sinne des Public Engagement gelten kann (vgl. Jünger und Fähnrich 2020, S. 392-393). Daher wurde nicht nur die wissenschaftsexterne Kommunikation mit Laien und/oder wissenschaftsexternen Expert*innen, sondern auch die private und berufliche Nutzung von Social Media erfasst.

Folgende Antwortmöglichkeiten waren für die Spezifizierung des Nutzungszwecks gegeben:

- „für private Zwecke, um mit Freunden und Verwandten in Kontakt zu bleiben“, „um mich allgemein über politische, kulturelle und andere Themen des öffentlichen Lebens zu informieren“, ,um mich über öffentlich zugängliche Themen mit Bezug zur Wissenschaft oder meinem Fachgebiet zu informieren“ (private Nutzung); 
- „um in beruflicher Hinsicht mit anderen Wissenschaftler*innen zu kommunizieren“, „um in beruflicher Hinsicht mit Kolleg*innen aus meinem direkten beruflichen Umfeld zu kommunizieren“, „um in beruflicher Hinsicht mit Studierenden zu kommunizieren“(berufliche Nutzung);

- „um mit wissenschaftsexternen Expert*innen (z. B. Journalist*innen), um mit interessierten Laien über meine Forschung oder mein Forschungsgebiet zu kommunizieren" (wissenschaftsexterne Kommunikation).

\subsubsection{Einflussfaktoren}

Beruflicher Kontext Um die Rahmenbedingungen der Wissenschaftler*innen zu erfassen, wurde gefragt, welchem Fachbereich sich die Befragten zuordnen und in welcher Funktion sie derzeitig an der Hochschule tätig sind (Doktorand*in und/oder wissenschaftliche/r Mitarbeiter*in; Postdoktorand*in; Akademische/r Rat/Rätin; Privatdozent*in; Juniorprofessor*in; Professor*in; nichtwissenschaftliches Personal; studentische/wissenschaftliche Hilfskraft). Die Befragten sollten zudem angeben, an welcher Universität sie beschäftigt sind. Sodann wurden nach Metag und Schäfer (2017) unterschieden, ob die Hochschulen zu den Social-Media-Experten, Allroundern, Mainstream, Traditionellen oder den Verweigerern gehören (institutioneller Kontext).

Disziplinzugehörigkeit Zusätzlich haben wir die Disziplin (Geistes- und Sozialwissenschaft, Lebenswissenschaft, Naturwissenschaft, Ingenieurswissenschaft) erfasst. Weiterhin sollten die Befragten angeben, inwiefern sie ihren Forschungsbereich als kompetitiv einschätzen und ob der Schwerpunkt ihrer Tätigkeit auf Grundlagenforschung, angewandter Forschung oder beidem gleichermaßen liegt.

Persönlichkeitsprädispositionen Um persönlichkeitsrelevante Faktoren wie die Einstellungen zur Social-Media-Nutzung zu analysieren, wurden von Pscheida et al. (2014, S. 44-45) drei Einflussfaktoren entnommen und über eine fünfstufige Likert-Skala (von „stimme gar nicht zu“ bis ,stimme voll und ganz zu“) abgefragt: Curiosity (vgl. auch Kashdan und Fincham 2004), Privacy Concerns (vgl. auch $\mathrm{Xu}$ et al. 2011) und Computer Anxiety (vgl. auch Venkatesh und Bala 2008). Die interne Konsistenz der jeweiligen Skalen wurden mit Cronbachs Alpha geprüft. $\mathrm{Cu}-$ riosity (Cronbachs Alpha=0,861) bezieht sich mit vier Items auf eine technikaffine Neugierde in Bezug auf technische Neuerungen: „Ich informiere mich so häufig wie möglich über technische Neuheiten, wie z. B. Smartphones, Computer, Software, Internet-Applikationen“, „Ich überlege häufig, wie ich technische Neuheiten für mich nutzen kann“, ,Wenn mich eine technische Neuheit interessiert, dann informiere ich mich gründlich darüber“, „Freunde oder Bekannte beschreiben mich als technikinteressiert“. Privacy Concerns benennt mit vier Items Sorgen um Daten und Nutzungsbedingungen: „Ich mache mir Sorgen, dass Daten, die ich im Internet bereitstelle, missbraucht werden könnten“, „Ich stelle ungern Informationen ins Internet, weil ich nicht weiß, was Andere mit ihnen machen könnten“, „Ich veröffentliche ungern Daten im Internet, weil diese in einer Weise verwendet werden 
könnten, die ich nicht vorhersehen kann“, „Ich kenne die Nutzungsbedingungen der Social-Media-Kanäle, die ich nutze“. Computer Anxiety bezieht sich ebenfalls mit vier Items konkret auf Ängste im Umgang mit Social Media: „Es macht mich nervös, mit Social Media zu arbeiten“, „Der Gedanke, dass es beim fehlerhaften Gebrauch von Social Media zu Datenverlust kommen könnte, ängstigt mich“, „Wenn ich Social Media nutze, habe ich Angst, Fehler zu machen, die ich nicht mehr korrigieren kann“, „Social Media wirken auf mich etwas einschüchternd“. Privacy Concerns und Computer Anxiety luden auf einen Faktor, daher wurden die beiden Konstrukte als Computer Anxiety zusammengefasst (Cronbachs Alpha=0,740). Im Folgenden unterscheiden wir diese Konstrukte als Technikaffinität und Technikabneigung.

Zudem wurden mit der Big-5-Kurzskala (BFI-10) von Rammstedt et al. (2013) die Persönlichkeitsdimensionen Extraversion, Verträglichkeit, Gewissenhaftigkeit, Neurotizismus und Offenheit gemessen.

\subsection{Auswahl und Stichprobe}

Aufgrund der zu vermutenden Einflussfaktoren ergaben sich als Anforderungen an die Grundgesamtheit zwei Kriterien: (1) Wissenschaftler*innen aus unterschiedlichen Disziplinen und (2) Berücksichtigung von jungen Wissenschaftler*innen (Doktoranden und PostDocs). Eine Rekrutierung über Publikationen (vgl. Allgaier et al. 2013a; Van Noorden 2014) oder Fachgruppen (vgl. Dudo und Besley 2016) war ungeeignet, da vor allem junge Wissenschaftler*innen dort möglicherweise noch nicht so präsent sind. Pscheida et al. (2014, S. 5) rekrutierten Wissenschaftler*innen an Hochschulen und außeruniversitären Forschungseinrichtungen in Deutschland über die Kontaktaufnahme mit den Presse- und Öffentlichkeitsabteilungen, sowie Rektorate und Prorektorate der Hochschulen und Forschungseinrichtungen. Ihre Bereitschaft zur Teilnahme erklärten allerdings nur knapp 25\% der angefragten Hochschulen, weshalb es sinnvoller erschien, diese Methode zu adaptieren, aber die Anzahl der Universitäten zu beschränken und zusätzlich zu den Rektoraten die Dekanate, Dekanatssekretariate sowie Institute und Institutssekretariate anzuschreiben. Für diese Studie kamen daher in einem bewussten Auswahlverfahren mit der Auswahl typischer Fälle Hochschulen in Betracht, die sich nach Forschungsprofilen bzw. Selbstbeschreibungen als deutsche Volluniversitäten bezeichnen. Laut Definition der Hochschulrektorenkonferenz gelten als ,,Volluniversität“ Hochschulen, die „die gesamte Breite der Natur- und Geisteswissenschaften berücksichtigen“ (Hochschulrektorenkonferenz 2020) und keinen (profilierenden) einzelnen Schwerpunkt aufweisen. Weiterhin achteten wir darauf, dass von jedem Kommunikationstyp (vgl. Metag und Schäfer 2017) mindestens eine Hochschule vertreten ist. 21 deutsche Universitäten erfüllten diese Kriterien. Im ersten Schritt wurden nur die Fakultätsdekane und die Dekanatssekretariate der 21 Hochschulen angeschrieben, im zweiten Schritt und nach einer Laufzeit von 13 Tagen kontaktierten wir für Universitäten, die eine geringe Response Rate aufwiesen, zusätzlich die Institute und verschickten eine Erinnerungsmail an die Dekanate aller Universitäten.

Insgesamt sind an den ausgewählten Hochschulen ca. 84.000 Wissenschaftler*innen beschäftigt. Da unklar bleibt, welche und wie viele Mitarbeiter*innen die Aufforderung zur Teilnahme durch ihre Fakultäten und Institute erhalten ha- 
ben, kann eine genaue Grundgesamtheit nicht bestimmt werden. Jedoch ging es uns darum, hinsichtlich der Einflussfaktoren ein möglichst breites Spektrum an Wissenschaftler*innen in Bezug auf Fachbereiche, Qualifikationsstufen, Alter und Geschlecht zu erfassen.

\section{Ergebnisse}

\subsection{Deskription der Stichprobe}

Insgesamt konnten über den Erhebungszeitraum im Juli 20191606 Datensätze gewonnen werden, 1431 Befragte nutzten dabei die deutsche und 175 Befragte die englische Version des Fragebogens. Von den 1606 Datensätzen wurden 1128 Fragebögen abgeschlossen (1016 deutsch, 112 englisch). Das Durchschnittsalter der Befragten ist 37 Jahre $(M=37,0 ; \mathrm{SD}=11,4)$, wobei die/der jüngste Teilnehmer*in 22 und die/der älteste Teilnehmer*in 78 Jahre alt ist. Hinsichtlich des Geschlechts ist das Sample relativ ausgewogen, etwa $50 \%$ der Befragten sind männlich und circa $48 \%$ weiblich. Die größte Gruppe innerhalb der Stichprobe bestreitet der wissenschaftliche Mittelbau. Studierende, Auszubildende oder technische Angestellte wurden sowie das nicht-wissenschaftlichen Personal aus dem Datensatz ausgeschlossen, so dass die Stichprobe insgesamt 1028 gültige Fälle enthält. In Bezug auf die Fachbereiche sind Lebens- und Naturwissenschaftler im Sample zu je etwa $23 \%$ vertreten, während Ingenieurswissenschaftler mit elf Prozent den kleinsten und Geistes- und Sozialwissenschaftler mit $42 \%$ den größten Anteil am Sample stellen. Von den Befragten, die entweder ausschließlich in der Forschung (41\% des Samples) oder in Forschung und Lehre gleichermaßen (51\%) tätig sind, gaben $36 \%$ an, in der Grundlagenforschung tätig zu sein. Etwa ein Drittel ist in der angewandten Forschung und ein weiteres Drittel in der angewandten Forschung und der Grundlagenforschung gleichermaßen tätig. Dabei schätzen $42 \%$ der Wissenschaftler*innen ihr Forschungsgebiet als (eher) nicht wettbewerbsorientiert ein, 29\% fanden, ihr Forschungsgebiet sei gleich wettbewerbsorientiert zu anderen Forschungsgebieten, und ebenfalls $29 \%$ hielten ihr Forschungsgebiet für (eher) wettbewerbsorientiert.

\subsection{Social-Media-Nutzung}

Bei Betrachtung der Nutzungshäufigkeiten der unterschiedlichen Social-MediaKanäle zeigt sich, dass YouTube der am meisten genutzte Kanal ist (ca. 94\% der Befragten nutzen YouTube mindestens selten). Facebook ist das am zweithäufigsten genutzte Medium in der Auswahl: Knapp 60\% der Befragten nutzen Facebook mindestens selten. Auch Podcasts $(60 \%)$ und Blogs $(58 \%)$ werden von mehr als der Hälfte der Befragten genutzt, Twitter von $37 \%$ der Wissenschaftler*innen mindestens selten. Instagram befindet sich mit etwa $35 \%$ in der Nutzungshäufigkeit nur knapp hinter dem Microblogging-Dienst. Weit abgeschlagen folgt mit nur knapp $6 \%$ Snapchat. Wenig überraschend, sind die Gründe für die Nutzung je nach Social Media unterschiedlich. Tab. 1 zeigt die kumulierten Prozente einer wöchentlichen Nutzung oder häufiger (wöchentlich, mehrmals wöchentlich, täglich). Entweder 
Tab. 1 Gründe zur Nutzung von Social Media

\begin{tabular}{|c|c|c|c|c|c|c|c|}
\hline & YouTube & Facebook & Twitter & Instagram & Snapchat & $\mathrm{B} \log \mathrm{s}$ & $\overline{\text { Podcasts }}$ \\
\hline Private Nutzung & 23,0 & 60,5 & 16,8 & 65,4 & 25,4 & 5,5 & 9,2 \\
\hline Informationen allgemein & 45,3 & 43,1 & 55,1 & 47,5 & - & 30,7 & 36,8 \\
\hline $\begin{array}{l}\text { Informationen zu Wissen- } \\
\text { schaft oder zur eigenen } \\
\text { Forschung }\end{array}$ & 29,7 & 25,9 & 45,4 & 15,3 & - & 26,8 & 23,8 \\
\hline $\begin{array}{l}\text { Kommunikation mit ande- } \\
\text { ren Wissenschaftler*innen }\end{array}$ & 2,2 & 8,2 & 23,5 & 4,0 & - & 5,3 & 1,6 \\
\hline $\begin{array}{l}\text { Kommunikation mit Kol- } \\
\text { leg*innen aus dem berufli- } \\
\text { chen Umfeld }\end{array}$ & 1,3 & 8,7 & 17,4 & 5,4 & - & 5,8 & 0,8 \\
\hline $\begin{array}{l}\text { Kommunikation mit Stu- } \\
\text { dierenden }\end{array}$ & 1,4 & 3,3 & 6,6 & 2,7 & - & 1,9 & 1,6 \\
\hline $\begin{array}{l}\text { Kommunikation mit } \\
\text { wissenschaftsexternen } \\
\text { Expert*innen über For- } \\
\text { schung }\end{array}$ & 1,7 & 4,2 & 16,1 & 1,9 & - & 4,3 & 2,0 \\
\hline $\begin{array}{l}\text { Kommunikation mit Laien } \\
\text { über Forschung }\end{array}$ & 1,9 & 5,1 & 14,5 & 4,0 & - & 4,2 & 2,5 \\
\hline$n$ & 598 & 645 & 392 & 373 & 63 & 623 & 642 \\
\hline
\end{tabular}

Kumulierte Prozente: Nutzung wöchentlich und häufiger

überwiegt die private Nutzung, um mit Freunden und Verwandten in Kontakt zu bleiben (Facebook, Instagram, Snapchat), oder die Nutzung, um sich allgemein über politische und kulturelle Themen sowie über spezifische Wissenschaftsthemen zu informieren (Blogs, Twitter, YouTube, Podcasts). Snapchat wird wöchentlich oder häufiger lediglich für private Zwecke genutzt. Zur Wissenschaftskommunikation werden die Social-Media-Angebote insgesamt eher selten genutzt. Eine Ausnahme ist hier Twitter: Um mit wissenschaftsexternen Expert*innen (16,1\%) oder interessierten Laien zu kommunizieren (14,5\%), ist Twitter der Social-Media-Kanal, der am häufigsten genutzt wird. Facebook oder Blogs werden zur Kommunikation mit wissenschaftsexternen Expert*innen nur noch von knapp $4 \%$ genutzt, ähnlich wie die Kommunikation mit Laien (Facebook: 5,1\%; Blogs: 4,2\%; hier auch Instagram: $4,0 \%)$. Eine Kommunikation über Podcasts oder YouTube findet nur bei weniger als 2,5\% der Befragten statt.

\subsection{Einflussfaktoren}

Im nächsten Schritt wird mit einer binär logistischen Regression geprüft, welche Einflussfaktoren die Social-Media-Nutzung von Wissenschaftler*innen erklären können. Als Prädiktoren haben wir die oben genannten Variablen in jeweils drei Blöcken persönliche Eigenschaften, Disziplinzugehörigkeit, beruflicher Kontext aufgenommen. Als abhängige Variable werden die Items, die sich auf eine berufliche Kommunikation der Wissenschaftler*innen (mit Kolleg*innen, mit anderen Wissenschaftler*innen) oder auf die eher nach außen gerichtete Kommunikation (mit Studierenden, wissenschaftsexternen Expert*innen, Laien) wie in Abschn. 4.1.1 
beschrieben zusammengefasst. Die interne Konsistenz der jeweiligen Konstrukte wurden mit Cronbachs Alpha geprüft und als ausreichend reliabel eingeschätzt (Facebook-Nutzung beruflich: Cronbachs Alpha=0,753; Facebook-Nutzung Wissenschaftskommunikation: Cronbachs Alpha=0,787; Twitter-Nutzung beruflich: Cronbachs Alpha=0,818; Twitter-Nutzung Wissenschaftskommunikation: Cronbachs Alpha=0,828). Da, wie oben bereits gezeigt, lediglich Twitter und Facebook für Wissenschaftskommunikation verwendet werden, werden im Folgenden auch nur die Regressionen für diese beiden Social-Media-Kanäle erstellt.

Die Tab. 2 zeigt die Regression auf die abhängige Variable der Nutzung von Facebook für die berufliche und wissenschaftsexterne Kommunikation. Für die berufliche Kommunikation zeigt sich, dass tendenziell Frauen $(\mathrm{B}=0,499, p=0,048)$ und ältere Personen Facebook nutzen $(B=0,046, p=0,005)$. Die Arbeit in der angewandten Forschung hat einen negativen Koeffizienten $(\mathrm{B}=-0,725, p=0,008)$. Stattdessen scheint die Einschätzung der Kompetitivität des Forschungsfeldes eher ausschlaggebend zu sein $(\mathrm{B}=0,320, p=0,004)$. Andere Variablen wie die Qualifikationsstufe, Persönlichkeitsprädispositionen oder auch Qualifikationsstufe haben keinen Einfluss auf die abhängige Variable. Mit jedem weiteren Variablenblock erhöht sich die Erklärleistung des Modells auf $\mathrm{R}^{2}=0,166$ (Nagelkerke).

Das Modell der Facebook-Nutzung in Bezug auf die Kommunikation mit z.B. wissenschaftsexternen Expert*innen und Laien hat mit einem Nagelkerke $\mathrm{R}^{2}=0,198$ eine etwas stärkere Erklärkraft als das vorherige Modell für die berufliche Kommunikation. Geschlecht hat diesmal keinen Einfluss, jedoch wieder geringfügig das Alter $(\mathrm{B}=0,047 ; p=0,011)$. In diesem Modell wird auch Extraversion signifikant $(\mathrm{B}=0,305 ; p=0,037)$. Personen, die eher extrovertiert sind, nutzen Facebook also eher für die nach außen gerichtete Kommunikation. Erneut wird auch die Wettbewerbsorientierung signifikant $(B=0,410 ; p=0,002)$. Wird das eigene Feld eher kompetitiv eingeschätzt, ist die Nutzung von Facebook für nach außen gerichtete Kommunikation wahrscheinlicher. Alle anderen Prädiktoren haben in diesem Modell keinen Einfluss.

Tab. 3 zeigt die Regression auf die berufliche Kommunikation $\left(\mathrm{R}^{2}=0,229\right.$; Nagelkerke) sowie die wissenschaftsexterne Kommunikation $\left(\mathrm{R}^{2}=0,22\right.$; Nagelkerke) von Twitter. Zwei Aspekte scheinen für die berufliche Kommunikation ausschlaggebend zu sein: Zum einen die Qualifikationsstufe ,Juniorprofessor*in“ oder „Privatdozent*in“ $(\mathrm{B}=1,858 ; p=0,047)$ und zum anderen erneut die Kompetitivität des Forschungsfeldes $(B=0,426 ; p=0,001)$. Diese Personengruppe tendiert daher eher als andere zu einer beruflichen Kommunikation auf Twitter mit anderen Kolleg*innen und/oder Wissenschaftler*innen.

Ähnlich wie bei der beruflichen Kommunikation ist auch für die nach außen gerichtete Kommunikation auf Twitter mit zum Beispiel Laien und wissenschaftsexternen Expert*innen erneut die Kompetitivität ein relevanter Faktor $(B=0,34$; $p=0,010)$. Die Arbeit in der Grundlagenforschung ist hier signifikant, jedoch mit einem negativen Koeffizienten $(\mathrm{B}=-0,707 ; p=0,036)$. Wer in diesem Feld arbeitet, nutzt Twitter eher nicht für die externe Kommunikation. 
Tab. 2 Einflussfaktoren bei der Nutzung von Facebook zur beruflichen und zur wissenschaftsexternen Kommunikation

\begin{tabular}{|c|c|c|c|c|c|c|c|}
\hline \multicolumn{2}{|c|}{ Facebook-Nutzung } & \multicolumn{3}{|c|}{ Berufliche Kommunikation } & \multicolumn{3}{|c|}{$\begin{array}{l}\text { Wissenschaftsexterne Kommuni- } \\
\text { kation }\end{array}$} \\
\hline & & $\mathrm{B}$ & SE & Sig & B & SE & Sig \\
\hline \multirow{10}{*}{$\begin{array}{l}- \\
\text { Block } \\
1\end{array}$} & Konstante & $-3,286$ & 1,742 & 0,051 & $-5,466$ & 2,065 & 0,08 \\
\hline & $\begin{array}{l}\text { Geschlecht } \\
\text { (1= weiblich) }\end{array}$ & 0,499 & 0,252 & 0,048 & 0,053 & 0,308 & 0,864 \\
\hline & Alter & 0,046 & 0,016 & 0,005 & 0,047 & 0,019 & 0,011 \\
\hline & Technikaffinität & $-0,213$ & 0,112 & 0,058 & $-0,116$ & 0,135 & 0,39 \\
\hline & Technikabneigung & $-0,278$ & 0,170 & 0,102 & $-0,369$ & 0,204 & 0,071 \\
\hline & B5: Extraversion & 0,208 & 0,119 & 0,081 & 0,305 & 0,146 & 0,037 \\
\hline & B5: Verträglichkeit & $-0,158$ & 0,131 & 0,231 & $-0,039$ & 0,161 & 0,808 \\
\hline & $\begin{array}{l}\text { B5: Gewissenhaftig- } \\
\text { keit }\end{array}$ & 0,025 & 0,158 & 0,873 & $-0,009$ & 0,196 & 0,964 \\
\hline & B5: Neurotizismus & 0,24 & 0,124 & 0,052 & 0,25 & 0,149 & 0,094 \\
\hline & B5: Offenheit & 0,168 & 0,132 & 0,204 & 0,255 & 0,169 & 0,131 \\
\hline \multirow[t]{7}{*}{$\begin{array}{l}\text { Block } \\
2\end{array}$} & $\begin{array}{l}\text { Geistes- und Sozial- } \\
\text { wissenschaften }\end{array}$ & 0,133 & 0,417 & 0,749 & $-0,112$ & 0,496 & 0,821 \\
\hline & Lebenswissenschaften & 0,291 & 0,438 & 0,506 & 0,462 & 0,513 & 0,367 \\
\hline & Naturwissenschaften & 0,026 & 0,435 & 0,953 & $-0,614$ & 0,549 & 0,264 \\
\hline & $\begin{array}{l}\ldots \text { in der Forschung } \\
\text { tätig }\end{array}$ & $-0,191$ & 0,228 & 0,402 & $-0,226$ & 0,284 & 0,426 \\
\hline & $\begin{array}{l}\ldots \text { in der Grundlagen- } \\
\text { forschung tätig }\end{array}$ & $-0,392$ & 0,278 & 0,159 & $-0,537$ & 0,338 & 0,112 \\
\hline & $\begin{array}{l}\ldots \text { in der angewandten } \\
\text { Forschung tätig }\end{array}$ & $-0,725$ & 0,273 & 0,008 & $-0,514$ & 0,318 & 0,106 \\
\hline & Kompetitivität & 0,32 & 0,112 & 0,004 & 0,41 & 0,134 & 0,002 \\
\hline \multirow{7}{*}{$\begin{array}{l}\text { Block } \\
3\end{array}$} & Doktorand*in & 0,867 & 0,619 & 0,161 & 0,988 & 0,714 & 0,166 \\
\hline & $\begin{array}{l}\text { PostDoc, Aka- } \\
\text { demischer Rat/ } \\
\text { Akademische Rätin }\end{array}$ & 0,839 & 0,604 & 0,165 & 1,11 & 0,701 & 0,113 \\
\hline & $\begin{array}{l}\text { Juniorprofessor*in, } \\
\text { Privatdozent*in }\end{array}$ & $-0,036$ & 0,713 & 0,959 & 1,16 & 0,791 & 0,143 \\
\hline & $\begin{array}{l}\text { Hochschule: Social- } \\
\text { Media-Spezialisten }\end{array}$ & $-0,084$ & 0,902 & 0,926 & $-0,068$ & 1,051 & 0,948 \\
\hline & $\begin{array}{l}\text { Hochschule: Allroun- } \\
\text { der }\end{array}$ & $-0,909$ & 0,878 & 0,3 & $-0,501$ & 1,021 & 0,624 \\
\hline & $\begin{array}{l}\text { Hochschule: Main- } \\
\text { stream }\end{array}$ & $-0,379$ & 0,897 & 0,673 & $-0,277$ & 1,049 & 0,791 \\
\hline & $\begin{array}{l}\text { Hochschule: Traditio- } \\
\text { nelle }\end{array}$ & $-0,841$ & 0,930 & 0,366 & $-1,215$ & 1,121 & 0,278 \\
\hline- & Nagelkerke $\mathrm{R}^{2}$ & - & - & 0,166 & - & - & 0,198 \\
\hline
\end{tabular}


Tab. 3 Einflussfaktoren bei der Nutzung von Twitter zur beruflichen und wissenschaftsexternen Kommunikation

\begin{tabular}{|c|c|c|c|c|c|c|c|}
\hline \multicolumn{2}{|c|}{ Twitter-Nutzung } & \multicolumn{3}{|c|}{ Berufliche Kommunikation } & \multicolumn{3}{|c|}{$\begin{array}{l}\text { Wissenschaftsexterne Kommuni- } \\
\text { kation }\end{array}$} \\
\hline & & $\mathrm{B}$ & SE & Sig & B & SE & Sig \\
\hline- & Konstante & $-3,664$ & 1,969 & 0,063 & $-2,024$ & 1,952 & 0,3 \\
\hline \multirow[t]{9}{*}{$\begin{array}{l}\text { Block } \\
1\end{array}$} & $\begin{array}{l}\text { Geschlecht } \\
(1=\text { weiblich })\end{array}$ & 0,218 & 0,298 & 0,464 & 0,032 & 0,2 & 0,915 \\
\hline & Alter & 0,018 & 0,019 & 0,353 & 0,011 & 0,019 & 0,565 \\
\hline & Technikaffinität & 0,119 & 0,133 & 0,373 & 0,186 & 0,134 & 0,166 \\
\hline & Technikabneigung & $-0,263$ & 0,199 & 0,186 & $-0,255$ & 0,2 & 0,202 \\
\hline & B5: Extraversion & 0,142 & 0,137 & 0,302 & 0,09 & 0,138 & 0,513 \\
\hline & B5: Verträglichkeit & 0,091 & 0,165 & 0,582 & $-0,085$ & 0,165 & 0,606 \\
\hline & B5: Gewissenhaftigkeit & 0,099 & 0,191 & 0,605 & 0,032 & 0,193 & 0,867 \\
\hline & B5: Neurotizismus & 0,167 & 0,159 & 0,293 & 0,106 & 0,158 & 0,501 \\
\hline & B5: Offenheit & 0,217 & 0,166 & 0,19 & 0,207 & 0,17 & 0,222 \\
\hline \multirow[t]{7}{*}{$\begin{array}{l}\text { Block } \\
2\end{array}$} & $\begin{array}{l}\text { Geistes- und Sozial- } \\
\text { wissenschaften }\end{array}$ & 0,188 & 0,457 & 0,681 & $-0,044$ & 0,468 & 0,925 \\
\hline & Lebenswissenschaften & $-0,001$ & 0,508 & 0,999 & 0,285 & 0,514 & 0,579 \\
\hline & Naturwissenschaften & $-0,152$ & 0,465 & 0,744 & 0,214 & 0,469 & 0,648 \\
\hline & $\begin{array}{l}\text {... in der Forschung } \\
\text { tätig }\end{array}$ & 0,078 & 0,277 & 0,779 & $-0,096$ & 0,281 & 0,732 \\
\hline & $\begin{array}{l}\text {... in der Grundlagen- } \\
\text { forschung tätig }\end{array}$ & $-0,494$ & 0,334 & 0,139 & $-0,707$ & 0,337 & 0,036 \\
\hline & $\begin{array}{l}\text {... in der angewandten } \\
\text { Forschung tätig }\end{array}$ & $-0,328$ & 0,348 & 0,345 & $-0,355$ & 0,351 & 0,311 \\
\hline & Kompetitivität & 0,426 & 0,131 & 0,001 & 0,34 & 0,132 & 0,010 \\
\hline \multirow{7}{*}{$\begin{array}{l}\text { Block } \\
3\end{array}$} & Doktorand*in & $-0,24$ & 0,606 & 0,692 & $-0,615$ & 0,606 & 0,310 \\
\hline & $\begin{array}{l}\text { PostDoc, Aka- } \\
\text { demischer Rat/ } \\
\text { Akademische Rätin }\end{array}$ & 0,128 & 0,609 & 0,834 & $-0,087$ & 0,607 & 0,886 \\
\hline & $\begin{array}{l}\text { Juniorprofessor*in, } \\
\text { Privatdozent*in }\end{array}$ & 1,858 & 0,936 & 0,047 & 1,411 & 0,84 & 0,093 \\
\hline & $\begin{array}{l}\text { Hochschule: Social- } \\
\text { Media-Spezialisten }\end{array}$ & 0,131 & 0,884 & 0,883 & 0,317 & 0,887 & 0,721 \\
\hline & $\begin{array}{l}\text { Hochschule: Allroun- } \\
\text { der }\end{array}$ & $-0,264$ & 0,856 & 0,758 & $-0,197$ & 0,859 & 0,819 \\
\hline & $\begin{array}{l}\text { Hochschule: Main- } \\
\text { stream }\end{array}$ & $-0,022$ & 0,897 & 0,98 & $-0,187$ & 0,903 & 0,836 \\
\hline & $\begin{array}{l}\text { Hochschule: Traditio- } \\
\text { nelle }\end{array}$ & $-0,937$ & 0,943 & 0,32 & $-0,814$ & 0,948 & 0,390 \\
\hline- & Nagelkerke $\mathrm{R}^{2}$ & - & - & 0,229 & - & - & 0,22 \\
\hline
\end{tabular}




\section{Diskussion}

Ziel der Studie war es, den Einfluss unterschiedlicher Faktoren auf die Social-MediaNutzung unter anderem bei der (Wissenschafts-)Kommunikation von Wissenschaftler*innen zu untersuchen. Dabei zeigte sich, dass in der Regel die private Nutzung (Kommunikation mit Freunden, Bekannten etc.) überwiegt. Lediglich Facebook und Twitter wurden von mehr als fünf Prozent für die Kommunikation mit anderen Wissenschaftler*innen, Kolleg*innen oder für die Kommunikation mit wissenschaftsexternen Expert*innen und Laien genutzt. Auch Van Noorden (2014, S. 127-129) konnte zeigen, dass neben beruflichen Netzwerken Facebook und Twitter wahrgenommen und - im Fall von Twitter - im professionellen Kontext häufig von Wissenschaftler*innen genutzt werden, um Diskussionen zu verfolgen, wissenschaftliche Inhalte $\mathrm{zu}$ kommentieren und eigene Inhalte $\mathrm{zu}$ posten. Wir haben im Anschluss nur für Facebook und Twitter die Einflussfaktoren auf ihre Zusammenhänge mit der Nutzung überprüft und deren Erklärkraft mit einer binär-logistischen Regressionsanalyse getestet. In den Modellen zeigte sich, dass nur wenige Faktoren signifikant sind.

\subsection{Einfluss der Disziplinzugehörigkeit: Anwendungsbezug und Kompetitivität}

Bisherige qualitative Studien zeigten, dass die Nutzung von Social Media sich stark an der Disziplinzugehörigkeit orientiert (vgl. Scheu und Schedifka 2018, S. 207) und auch die Motivation zur Außenkommunikation im Allgemeinen durch das Forschungsfeld beeinflusst wird (vgl. u. a. Kyvik 2005, Bentley und Kyvik 2011, S. 55). Dies konnte für die vorliegende Studie allerdings nur bedingt bestätigt werden. Das Forschungsfeld, also die Zugehörigkeit zu Lebens-, Natur-, Ingenieurs-, Geistesoder Sozialwissenschaften, hat keinen Einfluss auf die Kommunikation per Twitter und Facebook. Es zeigte sich, dass weder der Tätigkeitsschwerpunkt (Lehre bzw. Forschung) noch die Tätigkeit in der Grundlagenforschung diesen Einfluss verstärkt. In diesem Punkt widersprechen die Ergebnisse der bisherigen Forschung zu digitalen Medien (vgl. Pscheida et al. 2014, S. 33) und zum Public Engagement von Wissenschaftler*innen (vgl. Bauer und Jensen 2011, S. 6). Eindeutig zeigte sich allerdings, dass die Einschätzung, ob das eigene Forschungsgebiet eher wettbewerbsorientiert ist oder nicht, einen Einfluss besitzt. Wird es als eher kompetitiv eingeschätzt, ist damit eher eine Kommunikation auf Facebook und Twitter verbunden. Damit stützen die Ergebnisse der quantitativen Studie allerdings nicht die Befunde der qualitativen Studie von Scheu und Schedifka (2018, S. 203).

\subsection{Kaum Einfluss des beruflichen Kontextes}

Nach den Modellen scheint die Qualifikationsstufe in der vorliegenden Stichprobe keinen wesentlichen Einfluss auf die Nutzung von Facebook und Twitter für die berufliche oder nach außen gerichtete Wissenschaftskommunikation zu haben. Allenfalls die Personengruppe der Juniorprofessor*innen und Privatdozent*innen nutzte eher Twitter zur beruflichen Kommunikation mit anderen Kolleg*innen und/ oder Wissenschaftler*innen. Auch hier zeigen sich eher Übereinstimmungen mit 
Studien zur allgemeinen Außenkommunikation als solche, die sich spezifisch mit der Social-Media-Nutzung beschäftigen (vgl. Poliakoff und Webb 2016; Bauer und Jensen 2011). Was die Social-Media-Aktivität der Hochschule betrifft, liegen keine signifikanten Zusammenhänge zwischen dem Social-Media-Typ der Hochschule und der Social-Media-Nutzung der dort beschäftigten Wissenschaftler*innen vor.

\subsection{Kaum Einfluss von Alter, kein Einfluss von Persönlichkeitsprädispositionen oder Geschlecht}

In Bezug auf das Alter bestätigen die Ergebnisse bereits bekannte Muster der SocialMedia-Nutzung eher nicht (vgl. Donk 2012; Allgaier et al. 2013b; Scheu und Schedifka 2018): Alter zeigte sich nur dann als signifikant, wenn Facebook untersucht wurde. In diesem Falle sind es nicht die jüngeren Wissenschaftler*innen, sondern die älteren, die Facebook zur beruflichen und wissenschaftsexternen Kommunikation nutzen. Möglicherweise liegt das daran, dass Facebook insbesondere in Deutschland zunehmend weniger genutzt wird und bei jüngeren Personen von anderen Kanälen abgelöst wurde (Meedia 2014). Das Geschlecht stellte sich nur im Modell der beruflichen Kommunikation auf Facebook als signifikant heraus. Davon abgesehen ist die Kommunikation sowohl im beruflichen als auch wissenschaftsexternen Kontext nicht vom Geschlecht abhängig. Die Argumentation von Pscheida et al. (2014, S. 46), dass Wissenschaftler sich stärker mit neuen Technologien beschäftigen würden als Wissenschaftlerinnen, kann daher nicht gestützt werden. Allerdings sind auch hier die Ergebnisse eher konsistent mit der Forschung zum Public Engagement von Wissenschaftler*innen im Allgemeinen als mit jener zur Social-Media-Nutzung (vgl. Bauer und Jensen 2011; Dudo 2012).

Der von Pscheida et al. (2014) gefundene Einfluss der „Computer Anxiety“ konnte ebenfalls nicht bestätigt werden. Technikaffinität oder -abneigung hatte keinen Einfluss. Dies gilt auch für die Big-Five-Persönlichkeitsfaktoren, wenn alle Variablen in das Modell eingeschlossen sind. Typische Variablen wie Extraversion sind in den Fällen eher nicht ausschlaggebend. Auch dass neurotisches Verhalten zu höherer Social-Media-Nutzung beitragen kann (vgl. Correa et al. 2010; Ryan and Xenos 2011; Hughes et al. 2012) konnte nicht bestätigt werden.

Die eher geringen R-Quadrat-Werte in allen Modellen legen nahe, dass die unabhängigen Variablen nicht in der Lage sind, die abhängigen Variablen ausreichend gut zu erklären. Somit bedarf es weiterer Forschung, die andere Faktoren der Social-Media-Nutzung wie zum Beispiel Ziel- und Zweckgebundenheit der Nutzung integrieren sollte, um ein differenzierteres Bild der Motivationen zur Nutzung oder auch Nicht-Nutzung von Social Media zeichnen zu können. Ferner konnte diese Studie erneut zeigen, dass sich die Nutzung von Social Media im Sinne einer Kommunikation mit anderen aktuell nur auf wenige Anwendungen überhaupt beschränkt (Twitter, Facebook).

Wenn König (2017) gefolgt werden soll, dass sich Wissenschaftler*innen im Sinne der dialogischen Wissenschaftsvermittlung generell mehr mit der Fachcommunity und der interessierten Öffentlichkeit auseinandersetzen sollen, dann muss herausgefunden werden, woran dieser Dialog scheitert. Fortschritte in der (Medien-)Technologie und die damit verbundene Diversifizierung von Kommunikationswegen erfor- 
dern die Entwicklung komplexerer Erklärungsansätze, als sie die derzeitigen Modelle des Public Understanding oder Public Engagement of Science bieten (vgl. Bucchi und Trench 2016, S. 163). Zwar wäre es möglich, dass Wissenschaftler*innen Social Media nur sehr eingeschränkt zum Zweck der (quasi-öffentlichen) Wissenschaftskommunikation nutzen, Schulungen, Workshops oder Seminare zu diesem Thema anzubieten, um Missverständnisse in Bezug auf die Nachteile von Social Media abzubauen (vgl. Collins et al. 2016, S. 8). Jedoch scheint es weniger daran zu liegen, dass Wissenschaftler*innen nicht wissen, wie sie mit Social Media umzugehen haben, sondern daran, dass weitere Einflussfaktoren die Motivation zur Nutzung beeinflussen. Eine Möglichkeit wäre, dass dies (viel mehr als bisher betrachtet) Motive der Nicht-Nutzung sind. Die in dieser Studie qualitativ über offene Antworten erhobenen Gründe für eine Nicht-Nutzung von Social Media könnten daher zum Beispiel für quantitative Analysen im Zusammenhang mit den hier getesteten Einflussfaktoren verwendet werden. So wird oftmals Zeit als Grund für die Nicht-Nutzung von Social Media angeführt. Andere Autoren konnten aber zum Beispiel zeigen, dass dieser Faktor für die allgemeine Außenkommunikation keine Rolle spielt (vgl. Poliakoff und Webb 2016, S. 254). In diesem Zusammenhang erscheint es insgesamt sinnvoll, verstärkt Faktoren zu betrachten, die die Außenkommunikation von Wissenschaftler*innen im Allgemeinen beeinflussen, und sich nicht auf jene zu beschränken, die speziell auf die Social-Media-Nutzung von Wissenschaftler*innen Einfluss nehmen. Poliakoff und Webb (2016) zählen hierzu zum Beispiel die Erfahrungen von Wissenschaftler*innen mit der eigenen Außenkommunikation (,,attitude“), das wahrgenommene Engagement von Kolleg*innen (,descriptive norms“) und, ob die Wissenschaftler*innen die Partizipation als selbstbestimmt wahrnehmen (,,perceived behavioral control“). Diese Einstellungen könnten sich als wesentlich einflussreicher auf die Social-Media-Nutzung erweisen als die hier untersuchten Faktoren. Ein weiterer Faktor, der in zukünftigen Studien berücksichtigt werden sollte, ist Nationalität (vgl. Bentley und Kyvik 2011).

Auch wenn diese Studie wie viele andere Untersuchungen in diesem Themenbereich in ihrer Aussagekraft limitiert ist und in erster Linie auf weitere Forschung verweisen muss (vgl. Davies und Hara 2017, S. 564), liefern die vorliegenden Ergebnisse doch wichtige Erkenntnisse und damit weitere Forschungsansätze zum Verständnis der komplexen Kommunikationsbeziehungen zwischen Wissenschaftler*innen und der Gesellschaft in Zeiten sich ständig wandelnder Kommunikationsmedien.

Funding Open Access funding enabled and organized by Projekt DEAL.

Open Access Dieser Artikel wird unter der Creative Commons Namensnennung 4.0 International Lizenz veröffentlicht, welche die Nutzung, Vervielfältigung, Bearbeitung, Verbreitung und Wiedergabe in jeglichem Medium und Format erlaubt, sofern Sie den/die ursprünglichen Autor(en) und die Quelle ordnungsgemäß nennen, einen Link zur Creative Commons Lizenz beifügen und angeben, ob Änderungen vorgenommen wurden.

Die in diesem Artikel enthaltenen Bilder und sonstiges Drittmaterial unterliegen ebenfalls der genannten Creative Commons Lizenz, sofern sich aus der Abbildungslegende nichts anderes ergibt. Sofern das betreffende Material nicht unter der genannten Creative Commons Lizenz steht und die betreffende Handlung nicht nach gesetzlichen Vorschriften erlaubt ist, ist für die oben aufgeführten Weiterverwendungen des Materials die Einwilligung des jeweiligen Rechteinhabers einzuholen. 
Weitere Details zur Lizenz entnehmen Sie bitte der Lizenzinformation auf http://creativecommons.org/ licenses/by/4.0/deed.de.

\section{Literatur}

Acatech - Deutsche Akademie der Technikwissenschaften, Nationale Akademie der Wissenschaften Leopoldina, \& Union der deutschen Akademien der Wissenschaften (2017). Social Media und digitale Wissenschaftskommunikation. Analyse und Empfehlungen zum Umgang mit Chancen und Risiken in der Demokratie. München: Nationale Akademie der Wissenschaften Leopoldina.

Ajzen, I. (1991). The theory of planned behavior. Organizational Behavior and Human Decision Processes, 50(2), 179-211.

Allgaier, J., Dunwoody, S., Brossard, D., Lo, Y.-Y., \& Peters, H.P. (2013a). Journalism and social media as means of observing the contexts of science. BioScience, 63(4), 284-287.

Allgaier, J., Peters, H. P., Brossard, D., Dunwoody, S., \& Lo, Y.-Y. (2013b). Neurowissenschaftler schätzen „neue Medien“ wie Blogs und Facebook in der öffentlichen Wissenschaftskommunikation noch als weniger bedeutsam ein als journalistische Medien. e-Neuroforum, 19(2), 75-78.

Bader, A., Fritz, G., Gloning, T., \& Baranauskaite, J. (2012). Digitale Wissenschaftskommunikation 2010-2011. Eine Online-Befragung. Giessen: Giessener Elektronische Bibliothek.

Bauer, M.W., \& Jensen, P. (2011). The mobilization of scientists for public engagement. Public Understanding of Science, 20(1), 3-11.

Bentley, P., \& Kyvik, S. (2011). Academic staff and public communication: a survey of popular science publishing across 13 countries. Public Understanding of Science, 20, 48-63.

Besley, J.C., Dudo, A., \& Yuan, S. (2018). Scientists' views about communication objectives. Public Understanding of Science, 27, 708-730.

Bonetta, L. (2007). Scientists enter the blogosphere. Cell, 129(3), 443-445.

Bucchi, M., \& Trench, B. (2016). Science communication and science in society: a conceptual review in ten keywords. Technoscienza, 7(2), 151-168.

Burns, T. W., O'Connor, D. J., \& Stocklmayer, S. M. (2003). Science communication: a contemporary definition. Public Understanding of Science, 12, 183-202.

Collins, K., Shiffman, D., \& Rock, J. (2016). How are scientists using social media in the workplace? PLoS ONE, 11(10), 1-10.

Correa, T., Hinsley, A. W., \& de Zúñiga, H. G. (2010). Who interacts on the Web? The intersection of users' personality and social media use. Computers in Human Behavior, 26(2), 247-253.

Davies, S. R., \& Hara, N. (2017). Public science in a wired world: how online media are shaping science communication. Science Communication, 39, 563-568.

Donk, A. (2012). Ambivalenzen der Digitalisierung. Neue Kommunikations- und Medientechnologien in der Wissenschaft. Münster: Verlagshaus Monsenstein und Vannerdat.

Dudo, A. (2012). Toward a model of scientists' public communication activity: the case of biomedical researchers. Science Communication, 35, 476-501.

Dudo, A., \& Besley, J.C. (2016). Scientists' prioritization of communication objectives for public engagement. PLoS ONE, 11(2), 1-18.

Focke, J. (2017). Wissenschaft auf Facebook. In P. Weingart, H. Wormer, A. Wenninger \& R.F. Hüttl (Hrsg.), Perspektiven der Wissenschaftskommunikation im digitalen Zeitalter (S. 214-219). Weilerswist: Velbrück Wissenschaft.

Freie Universität Berlin Zahlen und Fakten. https://www.fu-berlin.de/universitaet/leitbegriffe/zahlen/ index.html. Zugegriffen: 20. Aug. 2020.

Friedrichsmeier, A., Laukötter, E., \& Marcinkowski, F. (2015). Hochschul-PR als Restgröße. Wie Hochschulen in die Medien kommen und was ihre Pressestellen dazu beitragen. In H. Bonfadelli, M.S. Schäfer \& S. Kristiansen (Hrsg.), Wissenschaftskommunikation im Wandel (S. 128-151). Köln: von Halem.

Geipel, A. (2017). Die audiovisuelle Vermittlung von Wissenschaft auf YouTube. In P. Weingart, H. Wormer, A. Wenninger \& R. F. Hüttl (Hrsg.), Perspektiven der Wissenschaftskommunikation im digitalen Zeitalter (S. 259-268). Weilerswist: Velbrück Wissenschaft.

Geipel, A. (2018). Wissenschaft@ YouTube. In E. Lettkemann, R. Wilke \& H. Knoblauch (Hrsg.), Knowledge in Action. Neue Formen der Kommunikation in der Wissensgesellschaft (S. 137-163). Wiesbaden: Springer VS.

Hochschulrektorenkonferenz (2020). Hochschultypen. https://www.hrk.de/themen/hochschulsystem/ hochschulrecht/hochschultypen/. Zugegriffen: 20. Aug. 2020. 
Hughes, D. J., Rowe, M., Batey, M., \& Lee, A. (2012). A tale of two sites: Twitter vs. Facebook and the personality predictors of social media usage. Computers in Human Behavior, 28, 561-569.

Jünger, J., \& Fähnrich, B. (2020). Does really no one care? Analyzing the public engagement of communication scientists on Twitter. New Media and Society, 22, 387-408.

Kashdan, T. B., \& Fincham, F. D. (2004). Facilitating curiosity: a social and self-regulatory perspective for scientifically based interventions. In P. A. Linley \& S. Joseph (Hrsg.), Positive Psychology in Practice (S. 482-503). Hoboken: Wiley.

König, M. (2017). Wissenschaftlerinnen und Wissenschaftler, nutzt soziale Medien! https://www. wissenschaftskommunikation.de/wissenschaftlerinnen-und-wissenschaftler-nutzt-soziale-medien5941/. Zugegriffen: 20. Aug. 2020.

Könneker, C. (2017). Wissenschaftskommunikation in vernetzten Öffentlichkeiten. In H. Bonfadelli, B. Fähnrich, C. Lüthje, J. Milde, M. Rhomberg \& M.S. Schäfer (Hrsg.), Forschungsfeld Wissenschaftskommunikation (S. 453-476). Wiesbaden: Springer VS.

Kyvik, S. (2005). Popular science publishing and contributions to public discourse among university faculty. Science Communication, 26, 288-311.

Liu, D., \& Campbell, W. K. (2017). The big five personality traits, big two metatraits and social media: a meta-analysis. Journal of Research in Personality, 70, 229-240.

Lo, Y.-Y. (2016). Online communication beyond the scientific community-Scientists' use of new media in Germany, Taiwan and the United States to address the public. Berlin: Freie Universität Berlin. Unveröffentlichte Dissertation

Maasen, S., \& Wenninger, A. (2017). Schneller, bunter, informativer: Mit Social Media zu einer neuen Wissenschaftskommunikation? In P. Weingart, H. Wormer, A. Wenninger \& R. F. Hüttl (Hrsg.), Perspektiven der Wissenschaftskommunikation im digitalen Zeitalter (S. 297-330). Weilerswist: Velbrück Wissenschaft.

Mahrt, M., \& Puschmann, C. (2014). Science blogging: an exploratory study of motives, styles, and audience reactions. Journal of Science Communication, 13(3), 1-17.

Meedia (2014). In allen Altersgruppen: Deutsche kehren Facebook laut „Social-Media-Atlas“ verstärkt den Rücken. https://meedia.de/2020/04/03/in-allen-altersgruppen-deutsche-kehren-facebook-lautsocial-media-atlas-verstaerkt-den-ruecken/. Zugegriffen: 30. Aug. 2020.

Metag, J., \& Schäfer, M. S. (2017). Hochschulen zwischen Social Media-Spezialisten und Online-Verweigerern. Eine Analyse der Online-Kommunikation promotionsberechtigter Hochschulen in Deutschland, Österreich und der Schweiz. Studies in Communication I Media, 6(2), 160-195.

Van Noorden, R. (2014). Scientists and the social network. Nature, 512(7513), 126-129.

Perez, M. (2010). Web 2.0 im Einsatz für die Wissenschaft. Information, Wissenschaft \& Praxis, 61(2), $129-134$.

Poliakoff, E., \& Webb, T. L. (2016). What factors predict scientists' intentions to participate in public engagement of science activities? Science Communication, 29, 242-263.

Pscheida, D., Albrecht, S., Herbst, S., Minet, C., \& Köhler, T. (2014). Nutzung von Social Media und onlinebasierten Anwendungen in der Wissenschaft. Erste Ergebnisse des Science 2.0-Survey 2013 des Leibniz-Forschungsverbunds „Science 2.0“. Dresden: Technische Universität Dresden.

Rammstedt, B., Kemper, C. J., Klein, M.C., Beierlein, C., \& Kovaleva, A. (2013). A short scale for assessing the big five dimensions of personality: 10 item big five inventory (BFI-10). methoden, daten, analysen, 7(2), 233-249.

Rauchfleisch, A. (2017). Wissenschaft auf Twitter. In P. Weingart, H. Wormer, A. Wenninger \& R. F. Hüttl (Hrsg.), Perspektiven der Wissenschaftskommunikation im digitalen Zeitalter (S. 291-294). Weilerswist: Velbrück Wissenschaft.

Ryan, T., \& Xenos, S. (2011). Who uses Facebook? An investigation into the relationship between the Big Five, shyness, narcissism, loneliness, and Facebook usage. Computers in Human Behavior, 27, 1658-1664.

Schäfer, M.S. (2017b). Wissenschaftskommunikation Online. In H. Bonfadelli, B. Fähnrich, C. Lüthje, J. Milde, M. Rhomberg \& M.S. Schäfer (Hrsg.), Forschungsfeld Wissenschaftskommunikation (S. 275-296). Wiesbaden: Springer VS.

Schäfer, M.S. (2017a). Wissenschaftskommunikation ist Wissenschaftsjournalismus, WissenschaftsPR ... und mehr. https://www.wissenschaftskommunikation.de/wissenschaftskommunikation-istwissenschaftsjournalismus-wissenschafts-pr-und-mehr-3337/. Zugegriffen: 20. Aug. 2020.

Scheu, A. M., \& Schedifka, T. (2018). Wissenschaftskommunikation im Netz. Eine explorative Studie zur Nutzung webbasierter sozialer Kommunikationskanäle. In L. M. Hagen, C. Lüthje, F. Ohser \& C. Seifert (Hrsg.), Wissenschaftskommunikation. Die Rolle der Disziplinen (S. 177-212). BadenBaden: Nomos. 
Venkatesh, V., \& Bala, H. (2008). Technology acceptance model 3 and a research agenda on interventions. Decision Sciences, 39(2), 273-315.

Wenninger, A. (2016). Wissenschaftsblogs: zwischen gesellschaftlicher Kontextherstellung und Selbstbezüglichkeit. In D. Schiek \& C. Ullrich (Hrsg.), Qualitative Online-Erhebungen (S. 25-54). Wiesbaden: Springer VS.

wissenschaftskommunikation.de (o. J.). Snapchat. https://www.wissenschaftskommunikation.de/format/ snapchat/. Zugegriffen: 30. Aug. 2020.

Xu, H., Dinev, T., Smith, J., \& Hart, P. (2011). Information privacy concerns: linking individual perceptions with institutional privacy assurances. Journal of the Association for Information Systems, 12, $798-824$.

Anne Hennig ist Abteilungsleiterin für Marketing und IT bei der Blanche Steuerberatungsgesellschaft.

Dr. phil. Sarah Kohler arbeitet am Karlsruher Institut für Technologie am Department für Wissenschaftskommunikation. 